\title{
MONGE-AMPÈRE EQUATIONS AND BELLMAN FUNCTIONS: THE DYADIC MAXIMAL OPERATOR
}

\author{
L. SLAVIN, A. STOKOLOS, AND V. VASYUNIN
}

\begin{abstract}
We find explicitly the Bellman function for the dyadic maximal operator on $L^{p}$ as the solution of a Bellman PDE of Monge-Ampère type. This function has been previously found by A. Melas $[\mathrm{M}]$ in a different way, but it is our PDE-based approach that is of principal interest here. Clear and replicable, it holds promise as a unifying template for past and current Bellman function investigations.
\end{abstract}

\section{INTRODUCTION}

For a locally integrable function $g$ on $\mathbb{R}^{n}$ and a set $E \subset \mathbb{R}^{n}$ with $|E| \neq 0$, let $\langle g\rangle_{E}=$ $\frac{1}{|E|} \int_{E} g$ be the average of $g$ over $E$. Let $p>1$ and $q>1$ be conjugate exponents, i.e. $p^{-1}+q^{-1}=1$. Let $\varphi$ be a nonnegative locally $L^{p}$-function on $\mathbb{R}^{n}$. Fix a dyadic lattice $D$ on $\mathbb{R}^{n}$ and consider the dyadic maximal operator

$$
M \varphi(x)=\sup _{I \ni x ; I \in D}\langle\varphi\rangle_{I} .
$$

Following F. Nazarov and S. Treil [NT], we define the Bellman function for $M \varphi$

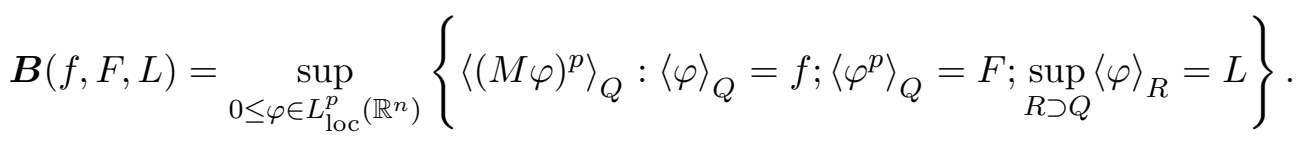

Observe that $\boldsymbol{B}$ is independent of $Q$ and well-defined on the domain

$$
\Omega=\left\{(f, F, L): 0<f \leq L ; f^{p} \leq F\right\} .
$$

Finding $\boldsymbol{B}$ will, among other things, provide a sharp refinement of the Hardy-LittlewoodDoob maximal inequality

$$
\|M \varphi\|_{p} \leq q\|\varphi\|_{p}
$$

In [NT], the authors show that $\boldsymbol{B}(f, F, L) \leq q^{p} F-p q f L^{p-1}+p L^{p}$, which implies (1.2). A. Melas in [M], using deep combinatorial properties of the operator $M$ and without relying on the Bellman PDE, finds $\boldsymbol{B}$ explicitly. In contrast, we develop a boundary value problem of Monge-Ampère type that $\boldsymbol{B}$ must satisfy (assuming sufficient differentiability) and solve it, producing the function from $[\mathrm{M}]$. Our approach has been used as the foundation of several recent Bellman function results. We first restrict our attention to the one-dimensional case and then show that the Bellman function does not depend on dimension.

2000 Mathematics Subject Classification. Primary 42A05, 42B35.

Key words and phrases. explicit Bellman function, Bellman PDE, Monge-Ampère equation, dyadic maximal operator.

L. Slavin's research supported in part by the NSF (grant DMS-0701254).

A. Stokolos' research supported in part by a grant from the Faculty Research and Development Fund of the College of Liberal Arts and Sciences of DePaul University.

V. Vasyunin's research supported in part by RFBR (grant 05-01-00925). 


\section{Finite-Differential and differential properties of $\boldsymbol{B}$}

Let $Q$ be an interval and $Q_{-}, Q_{+}$its left and right halves, respectively. Let $\left(f_{ \pm}, F_{ \pm}\right)=$ $\left(f_{Q_{ \pm}}, F_{Q_{ \pm}}\right), \quad(f, F)=\left(\left(f_{-}, F_{-}\right)+\left(f_{+}, F_{+}\right)\right) / 2$. Taking suprema in the identity

$$
\left\langle(M \varphi)^{p}\right\rangle_{Q}=\frac{1}{2}\left\langle(M \varphi)^{p}\right\rangle_{Q_{-}}+\frac{1}{2}\left\langle(M \varphi)^{p}\right\rangle_{Q_{+}}
$$

over all $\varphi$ with appropriate averages, we obtain

$$
\boldsymbol{B}(f, F, L) \geq \frac{1}{2} \boldsymbol{B}\left(f_{-}, F_{-}, \max \left\{f_{-}, L\right\}\right)+\frac{1}{2} \boldsymbol{B}\left(f_{+}, F_{+}, \max \left\{f_{+}, L\right\}\right) .
$$

Any function $B$ satisfying this pseudo-concavity property on $\Omega$ will be a majorant of the true Bellman function. The following theorem phrases this condition in a differential form.

Theorem 2.1. Let $z=(f, F)$. Assuming sufficient smoothness on the Bellman function $B$, condition (2.1) holds for all admissible triples if and only if

$$
\operatorname{det}\left(\frac{\partial^{2} B}{\partial z^{2}}\right)=0, B_{f f} \leq 0, B_{L} \geq 0 \text { on } \Omega ; \quad 2 B_{f L}+B_{L L} \leq 0, B_{L}=0 \text { when } f=L
$$

\section{Homogeneity, Boundary VAlue PRoblem, SOlution}

We reduce the order of the PDE in (2.2) by using the multiplicative homogeneity of $\boldsymbol{B}: \boldsymbol{B}(f, F, L)=L^{p} \boldsymbol{B}\left(f / L, F / L^{p}, 1\right) \stackrel{\text { def }}{=} L^{p} G(x, y)$, where $x=f / L, y=F / L^{p}$. In addition, $F=f^{p}$ only for functions that are constant on $Q$, so $\boldsymbol{B}\left(f, f^{p}, L\right)=L^{p}$, meaning $G\left(x, x^{p}\right)=1$. Coupling this with the first and the last conditions in (2.2), we get a boundary value problem for $G$ on the domain $\left\{(x, y) \mid 0<x \leq 1 ; x^{p} \leq y\right\}$ :

$$
G_{x x} G_{y y}=G_{x y}^{2} ; \quad G\left(x, x^{p}\right)=1 ; \quad p G(1, y)=G_{x}(1, y)+p y G_{y}(1, y) .
$$

We look for the solution of the Monge-Ampère equation (3.1) in the general parametric form

$$
G(x, y)=t x+f(t) y+g(t) ; \quad x+f^{\prime}(t) y+g^{\prime}(t)=0 .
$$

Fix a value of $t$, i.e. fix one of the straight-line trajectories in (3.2). Let $\left(u(t), u^{p}(t)\right)$ be the point where that trajectory intersects the lower boundary $y=x^{p}$. We have

$$
G\left(u, u^{p}\right)=t u(t)+f(t) u^{p}(t)+g(t)=1 ; \quad u(t)+f^{\prime}(t) u^{p}(t)+g^{\prime}(t)=0 .
$$

Differentiating the first equation and using the second one, we get, after some algebra, $f=-t /\left(p u^{p-1}\right), g=1-t u / q$. Assume now that the trajectory intersects the right boundary $x=1$ at the point $(1, v(t))$. Then $G(1, v)=t+f v+g$. On the other hand, parametrization (3.2) implies $G_{x}=t, G_{y}=f(t)$ and so the second boundary condition in (3.1) becomes $G(1, v)=\frac{t}{p}+f v$. This gives $g=-t / q$, allowing us to express $t=q /(u-1)$. Simplifying, we obtain a complete solution of the form (3.2):

$$
G(x, y)=\frac{y}{u^{p}} ; \quad x-\frac{q u-1}{q u^{p}} y-\frac{1}{q}=0 .
$$

In terms of the original variables, we get a Bellman function candidate near the boundary $f=L$ :

$$
B(f, F, L)=F u^{-p}\left(f / L, F / L^{p}\right)
$$




\section{From the CANDidate to the true FunCtion}

4.1. $B \geq \boldsymbol{B}$. One can readily verify that the rest of conditions (2.2) are satisfied by the candidate (3.4). Therefore, property (2.1) holds and one can perform the Bellman induction: take any non-negative function $\varphi \in L_{\text {loc }}^{p}\left(\mathbb{R}^{n}\right)$ and an interval $Q_{0} \in D$. For an interval $Q \subset Q_{0}, Q \in D$, let $X_{Q}=\left(f_{Q}, F_{Q}, L_{Q}\right)$ with $f, F$, and $L$ defined as in (1.1). Then

$$
\begin{aligned}
B\left(f_{Q_{0}}, F_{Q_{0}}, L_{Q_{0}}\right) & \geq \frac{1}{2} B\left(X_{\left(Q_{0}\right)_{-}}\right)+\frac{1}{2} B\left(X_{\left(Q_{0}\right)_{+}}\right) \\
& \geq \frac{1}{\left|Q_{0}\right|} \sum_{Q \subset Q_{0},|Q|=2^{-n}\left|Q_{0}\right|}|Q| B\left(X_{Q}\right) \geq \frac{1}{\left|Q_{0}\right|} \sum_{Q \subset Q_{0},|Q|=2^{-n}\left|Q_{0}\right|}|Q| L_{Q}^{p} \\
& =\frac{1}{\left|Q_{0}\right|} \sum_{Q \subset Q_{0},|Q|=2^{-n}\left|Q_{0}\right|}|Q|\left(\sup _{R \supset Q}\langle\varphi\rangle_{R}\right)^{p} \longrightarrow\left\langle(M \varphi)^{p}\right\rangle_{Q_{0}}, \text { as } n \rightarrow \infty .
\end{aligned}
$$

Here we have used that $B \geq L^{p}$. Taking supremum on the right over all $\varphi$ with the above $X_{Q_{0}}$ we get $B \geq \boldsymbol{B}$.

4.2. $B \leq \boldsymbol{B}$. To get the reverse inequality, we need to construct, for every point $(f, F, L) \in$ $\Omega$, a sequence of nonnegative functions on $(0,1),\left\{\varphi_{n}\right\}$, so that

$$
\lim _{n \rightarrow \infty}\left\langle\left(M \varphi_{n}\right)^{p}\right\rangle_{(0,1)} \geq B(f, F, L) .
$$

To do this, we use the trajectories $t=$ const of the Monge-Ampère equation from Section 3. In the original variables, this gives

$$
f=\frac{L}{q}+A F
$$

On the boundary $f=L$ going along these trajectories yields the extremal sequence

$$
\varphi_{n}(t)= \begin{cases}\alpha_{n} L & 0<t<2^{-n} \\ \varphi_{n}\left(2^{k} t-1\right) & 2^{-k}<t<2^{-k+1}, k=2, \ldots, n \\ \beta_{n} \varphi_{n}(2 t-1) & \frac{1}{2}<t<1 .\end{cases}
$$

The definition is understood recursively, whereby the function is defined on a portion of $(0,1)$, then on the same portion of the remaining part, and so on. The numbers $\alpha_{n}$ and $\beta_{n}$ are chosen so that $\left\langle\varphi_{n}\right\rangle_{(0,1)}=L$ and $\left\langle\varphi_{n}^{p}\right\rangle_{(0,1)}=F$. This means

$$
\frac{1}{2^{n}} \alpha_{n}+\frac{1}{2} \beta_{n}=\frac{1}{2^{n}}+\frac{1}{2} ; \frac{1}{2^{n}} \alpha_{n}^{p}+\frac{1}{2} \beta_{n}^{p} \frac{F}{L^{p}}=\left(\frac{1}{2^{n}}+\frac{1}{2}\right) \frac{F}{L^{p}} .
$$

One can show that $\alpha_{n} M \varphi_{n} \geq \varphi_{n}$ and $\alpha_{n} \rightarrow u\left(1, F / L^{p}\right)$ with $u$ defined by (3.3). Therefore,

$$
\lim _{n \rightarrow \infty}\left\langle\left(M \varphi_{n}\right)^{p}\right\rangle_{(0,1)} \geq \lim _{n \rightarrow \infty} \frac{1}{\alpha_{n}^{p}}\left\langle\varphi_{n}^{p}\right\rangle_{(0,1)}=\lim _{n \rightarrow \infty} \frac{F}{\alpha_{n}^{p}}=F u^{-p}\left(1, F / L^{p}\right)=\boldsymbol{B}(L, F, L),
$$

which gives $\boldsymbol{B}(L, F, L) \geq B(L, F, L)$.

On the boundary $F=f^{p}$ the situation is simple: here the only test functions are constants and so $B\left(f, f^{p}, L\right)=\boldsymbol{B}\left(f, f^{p}, L\right)=L^{p}$. Having constructed the extremal sequences on the two boundaries, we get the extremal sequence at any point $(f, F, L)$ with $f>L / q$ as their weighted dyadic rearrangement built along the unique extremal trajectory of the form (4.2) passing through the point.

One observes, however, that trajectories (4.2) cannot be used with $A<0$, since they then would intersect the "forbidden" boundary $f=0$. (It is forbidden because, for a nonnegative function, $f=0$ implies $F=0$. ) In fact, in the region $0<f<L / q$, no trajectory can lean either to the left or to the right (the forbidden boundary to the left, the existing extremal 
trajectory $f=L / q$ to the right). We conclude two things: the trajectories are vertical in this region and the candidate (3.4) no longer works there. However, this is quickly rectified: If $G(x, y)=a(x) y+b(x)$, then $G\left(x, x^{p}\right)=1$ implies that $G(x, y)=1+a(x)\left(y-x^{p}\right)$. Now $G_{x x} G_{y y}-G_{x y}^{2}=-\left(a^{\prime}(x)\right)^{2}=0$, and $G(1 / q, y)=q^{p} y$ implies that $a(x)=q^{p}$. Thus we get the unique two-piece Bellman function candidate

$$
B(f, F, L)= \begin{cases}F u^{-p}\left(f / L, F / L^{p}\right) & L<q f \\ L^{p}+q^{p}\left(F-f^{p}\right) & L \geq q f .\end{cases}
$$

(In the notation of $[\mathrm{M}], u^{-p}(x, y)=\omega_{p}((p x-p+1) / y)^{p}$.) This $B$ still satisfies (2.1). Therefore, Bellman induction (4.1) works. We now need an extremal sequence proving that $\boldsymbol{B} \geq B$ in the region $L \geq q f$. There is a unique extremal trajectory passing through each point of the region. However, the trajectory is vertical and so intersects the boundary of $\Omega$ at a single point; as a result we cannot use a weighted average of boundary extremal sequences like we just did for the region $L>f / q$. We deal with it by tilting the trajectory slightly to the right, which produces a (distant) second boundary point, at the boundary $f=L$. This lets us use the extremal sequence $\varphi_{n}$ from (4.3), while simultaneously reducing the tilt. Namely, fix $(f, F, L)$ and $k \geq 1$. Define $\gamma_{k}$ and $F_{k}$ so that $L-\gamma_{k}=2^{k}\left(f-\gamma_{k}\right)$ and $F_{k}-\gamma_{k}^{p}=2^{k}\left(F-\gamma_{k}^{p}\right)$. (Observe that $\gamma_{k} \rightarrow f$ and $F_{k} \rightarrow \infty$.) Using (4.3), form a sequence $\left\{\varphi_{k, n}\right\}_{n=1}^{\infty}$ with $\left\langle\varphi_{k, n}\right\rangle_{(0,1)}=L$ and $\left\langle\varphi_{k, n}^{p}\right\rangle_{(0,1)}=F_{k}$, so that $\left\langle\left(M \varphi_{k, n}\right)^{p}\right\rangle_{(0,1)} \rightarrow$ $B\left(L, F_{k}, L\right)$, as $n \rightarrow \infty$. Let

$$
\psi_{k, n}(t)= \begin{cases}\varphi_{k, n}\left(2^{k} t\right) & 0<t<2^{-k} \\ \gamma_{k} & 2^{-k}<t<1 \\ 2 L-f & 1<t<2\end{cases}
$$

Direct computation shows that $\left\langle\psi_{k, n}\right\rangle_{(0,1)}=f,\left\langle\psi_{k, n}^{p}\right\rangle_{(0,1)}=F$, and $\left\langle\psi_{k, n}\right\rangle_{(0,2)}=L$. Then

$$
\begin{aligned}
\left\langle\left(M \psi_{k, n}\right)^{p}\right\rangle_{(0,1)} & \geq L^{p}\left(1-2^{-k}\right)+2^{-k}\left\langle\left(M \varphi_{k, n}\right)^{p}\right\rangle_{(0,1)} \\
& \underset{n \rightarrow \infty}{\longrightarrow} L^{p}\left(1-2^{-k}\right)+2^{-k} B\left(L, F_{k}, L\right) \\
& \underset{k \rightarrow \infty}{\longrightarrow} L^{p}+\left(F-f^{p}\right) u^{-p}(1, \infty)=L^{p}+q^{p}\left(F-f^{p}\right),
\end{aligned}
$$

\section{SeVEral Dimensions}

It turns out that the Bellman function $(1.1),(4.4)$ is dimension-free. Fix a dyadic cube $Q$ and let $Q_{1}, \ldots, Q_{2^{n}}$ be its dyadic offspring. Then

$$
B\left(2^{-n} \sum_{k=1}^{2^{n}} z_{k}, L\right) \geq 2^{-n} \sum_{k=1}^{n} B\left(z_{k}, \max \left\{f_{k}, L\right\}\right) .
$$

Therefore, we can run the induction (4.1) to prove that $B \geq \boldsymbol{B}$. The other direction is shown by a trivial modification of the one-dimensional maximizing sequences. A similar argument can be used to show that the same Bellman function works for the maximal operator on trees, the setting of choice in $[\mathrm{M}]$.

\section{REFERENCES}

[M] A. Melas. The Bellman functions of dyadic-like maximal operators and related inequalities. Advances in Mathematics, Vol. 192 (2005), No. 2, pp. 310-340.

[NT] F. Nazarov, S. Treil. The hunt for Bellman function: applications to estimates of singular integral operators and to other classical problems in harmonic analysis. (Russian)Algebra i Analiz, Vol. 8 (1996), No. 5, pp. 32-162; translation in St. Petersburg Math. J., Vol. 8 (1997), No. 5, pp. 721-824. 
MONGE-AMPÈRE EQUATIONS AND BELLMAN FUNCTIONS: THE DYADIC MAXIMAL OPERATOR 5

University OF Missouri-Columbia

E-mail address: leonid@math.missouri.edu

DePaul University

E-mail address: astokolo@depaul.edu

St. Petersburg Department of the V. A. Steklov Mathematical Institute, Ras

E-mail address: vasyunin@pdmi.ras.ru 